\title{
Decentralized Polynomial Observer Design for Discrete-Time Large-Scale Polynomial T-S Fuzzy System
}

\author{
Seung-Hun Han, ${ }^{1}$ Van-Phong Vu $\mathbb{D}^{2},{ }^{2}$ and Manh-Son Tran ${ }^{2}$ \\ ${ }^{1}$ Mechanical System Engineering Department, Tongyeong Campus, Gyeongsang National University, 53064, Republic of Korea \\ ${ }^{2}$ Automatic Control Department, Ho Chi Minh City University of Technology and Education, 700000, Vietnam \\ Correspondence should be addressed to Van-Phong Vu; phongvv@hcmute.edu.vn
}

Received 19 November 2018; Revised 23 February 2019; Accepted 24 March 2019; Published 22 April 2019

Academic Editor: Alessandro Mauro

Copyright (c) 2019 Seung-Hun Han et al. This is an open access article distributed under the Creative Commons Attribution License, which permits unrestricted use, distribution, and reproduction in any medium, provided the original work is properly cited.

\begin{abstract}
In this paper, a new approach for synthesizing a decentralized observer is proposed to estimate the unmeasurable states of a discrete-time large-scale nonlinear system. The large-scale nonlinear system in this study is modeled in terms of a set of discretetime polynomial Takagi-Sugeno (T-S) fuzzy subsystems and interconnection terms. This modeling method will assist to reduce significantly the number of fuzzy rules. The interconnection terms are considered as the unknown inputs; then, the unknown input method is employed to design observer for this system. It should be emphasized that the interconnection parts in this paper are arbitrary and their effects are eliminated completely. On the basis of the Lyapunov methodology and SOS (Sum of Square) technique, the conditions for observer design expressed under the framework of SOS are derived in the main theorems. Finally, an illustrative example is presented to show the effectiveness and merit of the proposed method.
\end{abstract}

\section{Introduction}

Nowadays, due to the development of technology and human's demand, the complexity and large-scale of the system are significantly increasing. These complicated systems are called large-scale systems which consist of a set of interconnection subsystems. The large-scale system has appeared in many real applications such as communication systems, power systems, ecosystems, and transportation systems. There are plenty of studies focusing on stability analysis, controller design, and observer design for the large-scale nonlinear systems in recent years [1-4]. For example, the methods to design the decentralized controllers for the largescale nonlinear systems were investigated in $[1,2]$, where the interconnection terms of these systems were unknown and estimated by using neural networks. In [4], an optimal controller design method based on the $H_{\infty}$ technique was proposed for a large-scale nonlinear system to stabilize the system and deal with the impacts of disturbance and saturated actuator.

Recently, a method for modeling nonlinear systems called Takagi-Sugeno fuzzy system was introduced in [5] in which a nonlinear system was represented in terms of a set of
IF-THEN rules and linear subsystems. This method came out in 1984 and, until now, the T-S fuzzy model increasingly plays a very important role in modeling the nonlinear systems. Numerous studies focusing on the T-S fuzzy system could be found in books $[6,7]$. Moreover, the T-S fuzzy model is recently employed to represent the large-scale nonlinear system which is called "large-scale T-S fuzzy system". In the past few years, there have been a lot of papers concentrating on solving the issues of the large-scale T-S fuzzy system such as stability analysis, controller design, and observer synthesis [8-15]. In [8], an approach for designing controller was investigated for large-scale nonlinear systems in which the interconnection parts were with the nonlinear form. Moreover, a method for stability analysis and controller design based on $H_{\infty}$ was proposed for the discrete-time large-scale T-S fuzzy system [9] where the piecewise Lyapunov function was employed instead of the common Lyapunov function. In [11], a method that relied on $H_{\infty}$ technique for designing controller was studied for large-scale T-S fuzzy systems with the existence of both disturbance and uncertainties. However, the drawback of the modeling method based on the T-S fuzzy model is that when the number of subsystems or number of linearization terms of each subsystem increases, the number 
of fuzzy rules will exponentially grow up. This results in the increase of the computational load.

Recently, an extended form of the T-S fuzzy model called "polynomial T-S fuzzy model" was introduced in [16]. The major difference between the T-S fuzzy system and the polynomial T-S fuzzy system was that the elements of the system, input, and output matrices of the polynomial T-S fuzzy model were in polynomial form instead of the constant of the T-S fuzzy model. By putting the variables inside the system matrices, it will help to reduce the number of linearization terms and lead to significant decrease in the modeling error and number of fuzzy rules with respect to the T-S fuzzy model. With the aid of the Sum-of-Square (SOS) technique [17], the polynomial T-S fuzzy system becomes an increasingly popular tool for modeling nonlinear systems. In recent years, there have been a lot of researchers focusing on stability analysis, controller design, and observer synthesis for polynomial T-S fuzzy systems [18-20]. For example, in [18], observer and observer-based controller were synthesized for the polynomial T-S fuzzy system with three classifications. Moreover, a method to design a polynomial observer-based controller for the polynomial T-S fuzzy system was introduced in [19] in which the premise variables were unmeasurable and they need to be estimated as well. However, to the best of our knowledge, there is no previous study applying the polynomial T-S fuzzy framework for modeling discrete-time large-scale nonlinear system.

In practice, it is very difficult to measure some physic parameters of many practical systems. Moreover, in some cases, using the sensor to obtain the information of the variables is costly or encounters technical problems such as the impact of noise. However, these variables are necessary for monitoring system and designing controller. Therefore, replacing sensors by the observer is a pressing issue and plays a substantial role in the control field. Regarding observer synthesis for large-scale systems, many papers have been published in the past few years [21-29] focusing on solving this issue. For example, the decentralized $H \infty$ filters were synthesized for the nonlinear large-scale system and the nonlinear large-scale system with unknown interconnection terms in $[21,22]$, respectively. However, the interconnection term in [21] must be modeled in linear form and the interconnection part in [22] must satisfy the bounded constraints. Moreover, an approach to design a robust observer for a multimachine power system expressed in terms of nonlinear large-scale system was concerned in [23] in which the sliding mode technique was employed to eliminate the effect of the uncertainties and estimate the unknown states. In [25], an observer was synthesized to detect faults of the affine largescale T-S fuzzy system. It should be noted that the interconnection terms were unknown, but the interconnection terms in [25] have to fulfill the norm bounded constraints. Additionally, the filter and observer were synthesized for discretetime large-scale T-S fuzzy system in [26-28]. However, the interconnection terms in these papers must be expressed under the linear form of the T-S fuzzy model. Otherwise, the proposed methods in [26-29] failed to design the filter for these systems. In other words, there are two forms of the interconnection terms in the previous studies [21, 22, 25-29]: one is known and other is unknown. The advantage of the unknown interconnection terms is that we do not need to know exactly the form of interconnection terms and these terms do not need to linearize to the linear form or T-S fuzzy model in order to reduce the modeling error and complexity. However, the unknown interconnection terms must satisfy the specific constraints such as the bounded constrains in $[22,25]$. Regarding knowing interconnection terms, their advantage is that they are unnecessary to fulfill any constraint. However, they have to be able to model under the linear form or T-S fuzzy model [21, 26-29]; it leads to increase in the modeling error, complexity, and computational load during designing observer/controller for the large-scale systems.

From above discussions, it is easily seen that employing the T-S fuzzy model [8-15] for representing large-scale nonlinear system will cause to significantly increase the number of fuzzy rules and modeling errors and make the computational load heavier. In addition, the interconnection terms of the large-scale system $[22,25]$ must be bounded and in [21, 26-29] have to model under the T-S fuzzy model form. However, in practice, in some cases, the upper bound of the interconnection terms is not easy to determine and the interconnection terms of several systems are unable/difficult to be represented in the T-S fuzzy model; thus, the proposed methods in the previous papers are not applicable to these systems. Additionally, to the full extent of our knowledge, the polynomial T-S fuzzy model has not been applied to represent the discrete-time nonlinear system in any previous study. Due to these reasons, we are inspired to study further a new method for synthesizing a decentralized observer to estimate the unknown states of the discrete-time large-scale polynomial T-S fuzzy system with arbitrary interconnection terms. The main contributions of this paper are emphasized in the following aspects:

(1) This is the first time the polynomial T-S fuzzy system used to model the discrete-time large-scale nonlinear systems. This modeling method will help to reduce the number of fuzzy rules, modeling errors, and computational load with respect to large-scale T-S fuzzy systems in [8-15].

(2) In this paper, the interconnection terms are arbitrary and are treated as unknown inputs. The proposed approach allows us to apply for the large-scale systems in which the interconnection terms are not known exactly. In addition, the interconnection term in this paper is unnecessary to satisfy the bounded constraints which are mandatory in $[22,25]$. Moreover, with the proposed method, the interconnection terms do not need to linearize to transform them under the T-S fuzzy model form [21, 26-29], which assists to avoid increasing of the fuzzy rules and complexity of the modeled system. The unknown input method [30-34] is employed to design observer for the largescale polynomial T-S fuzzy system in which the effects of the interconnection terms are eliminated completely and unmeasurable states are estimated asymptotically. 
(3) The fuzzy Lyapunov function is applied in this paper instead of the common Lyapunov function to make the conditions for observer design to be more relaxed.

The rest of this paper is organized as follows. The considered system and the solved problem in this paper are presented in Section "System Model and Problem Description." The main theorems for designing a decentralized polynomial observer are shown in Section "Decentralized Observer Design". A numerical example to prove the effectiveness of the proposed method is mentioned in Section "Illustrative Example". Finally, several conclusions are stated in Section "Conclusion".

Notations. In this paper, $\Lambda>0(\Lambda<0)$ indicates the positive (negative) definite matrix. $\Lambda^{T}$ stands for the transpose of matrix $\Lambda . \Lambda^{-1}$ represent the inverse of matrix $\Lambda$ and $I$ indicates the identity matrix. $\Lambda^{+}$stands for the MoorePenrose pseudoinverse of $\Lambda$ where $\Lambda^{+}=\left(\Lambda^{T} \Lambda\right)^{-1} \Lambda^{T}$. (*) stands for the symmetric term of a matrix.

\section{System Model and Problem Description}

Consider the discrete-time large-scale nonlinear system with $N$ subsystems as follows.

Subsystem $l$

$$
\begin{aligned}
x_{l}(k+1) & =h^{l}\left(x_{l}(k), u_{l}(k)\right)+g_{l}(x) \\
y_{l}(k) & =C^{l} x_{l}(k) \\
l & =1,2, \ldots N
\end{aligned}
$$

where $x_{l}(k)$ and $u_{l}(k)$ are the vectors of the state variables and inputs of the $l$-th subsystem. $h^{l}\left(x_{l}(k), u_{l}(k)\right)$ is the nonlinear function and $g_{l}(x)$ is the interconnection term which expresses the interaction between $l$-th subsystem with others. $x$ is a vector of states which consist of all states $x_{l}(k)$.

Assumption 1. Assume that the interconnection part of system (1) satisfies the matching condition $g_{l}(x)=T^{l} \vartheta_{l}(x)$. The matrices $T^{l}$ and $C^{l}$ are full column and row rank, respectively. The rank of $\left(C^{l} T^{l}\right)$ is equal to the rank of $\left(T^{l}\right)$.

Remark 2. The rank conditions of matrices $T^{l}$ and $C^{l}$ in Assumption 1 are necessary to guarantee the existence of the general solution of matrix equation in the consequent section. This is a common condition that can be found in many previous papers studying about unknown input method such as [30-34].

On the basis of Assumption 1 and applying the sector nonlinear or linearization method in $[6,7]$, system (1) is transformed into the discrete-time large-scale polynomial TS fuzzy system as follows.

\section{Subsystem l-th}

Rule $i$. If $\theta_{1}^{l}(k)$ is $M_{i 1}^{l}, \ldots$, and $\theta_{s}^{l}(k)$ is $M_{i s}^{l}$, then

$$
\begin{aligned}
x_{l}(k+1)= & A_{i}^{l}\left(\zeta_{l}(k)\right) x_{l}(k)+B_{i}^{l}\left(\zeta_{l}(k)\right) u_{l}(k) \\
& +T^{l} \vartheta_{l}(x) \\
y_{l}(k)= & C^{l} x_{l}(k)
\end{aligned}
$$

in which $\theta^{l}(k)=\left[\theta_{1}^{l}(k), \theta_{2}^{l}(k), \ldots, \theta_{s}^{l}(k)\right]$ is a vector of the premise variables which is known in advance, $M_{i j}^{l}(i=$ $\left.1,2, \ldots, r_{l} ; j=1,2, \ldots, s\right)$ is the fuzzy set, and $r_{l}$ and $s$ are the number of fuzzy rules and premise variables, respectively.

Overall large-scale system (2) is represented.

Subsystem $l$

$$
\begin{aligned}
& x_{l}(k+1)=\sum_{i=1}^{r_{l}} \beta_{i}^{l}\left(\theta^{l}(k)\right) \\
& \cdot\left\{A_{i}^{l}\left(\zeta_{l}(k)\right) x_{l}(k)+B_{i}^{l}\left(\zeta_{l}(k)\right) u_{l}(k)+T^{l} \vartheta_{l}(x)\right\} \\
& y_{l}(k)=C^{l} x_{l}(k)
\end{aligned}
$$

where $A_{i}^{l}\left(\zeta_{l}(k)\right), B_{i}^{l}\left(\zeta_{l}(k)\right)$ are the time-varying system and input polynomial matrices with time-varying entry $\zeta_{l}(k) . T^{l}$ and $C^{l}$ are the interconnection and output matrices. $y_{l}(k)$ is the vector of the outputs. $\zeta_{l}(k)$ is the time-varying variables which could be output, external signal or time. $\beta_{i}^{l}\left(\theta^{l}(k)\right)$ is the normal weight function and fulfills the following conditions:

$$
\begin{aligned}
w_{i}^{l}\left(\theta^{l}(k)\right) & =\prod_{j=1}^{s} M_{i j}^{l}\left(\theta^{l}(k)\right), \\
w_{i}^{l}\left(\theta^{l}(k)\right) & \geq 0, \\
\beta_{i}^{l}\left(\theta^{l}(k)\right) & =\frac{w_{i}^{l}\left(\theta^{l}(k)\right)}{\sum_{i=1}^{r_{l}} w_{i}^{l}\left(\theta^{l}(k)\right)} \\
0 \leq \beta_{i}^{l}\left(\theta^{l}(k)\right) & \leq 1 \\
\text { and } \sum_{i=1}^{r_{l}} \beta_{i}^{l}\left(\theta^{l}(k)\right) & =1 .
\end{aligned}
$$

Suppose that some state variables of system (1) are unmeasurable and the interconnection term $g_{l}(x)$ is arbitrary and the upper bound constraints of $g_{l}(x)$ are unknown as well. However, these state variables are necessary to monitor the system. It should be noted that the interconnection term $g_{l}(x)$ will make the observer design much more difficult. In addition, the interconnection terms $g_{l}(x)$ are arbitrary and the upper bounds of $g_{l}(x)$ are not known; therefore, the methods in $[21,22,25-29]$ are unable to employ to design an observer for this system. Therefore, the objective of this study is to propose a new approach to synthesize a decentralized observer for estimating the unknown states and completely eliminate the influences of the interconnection terms. 


\section{Useful Lemmas}

Lemma 3. Considering two matrices $\Psi$ and $\Lambda$ with appropriate dimensions, the following condition holds for any positive scalar $\epsilon$ :

$$
\Psi^{T} \Lambda+\Lambda^{T} \Psi \leq \epsilon \Psi^{T} \Psi+\epsilon^{-1} \Lambda^{T} \Lambda
$$

Lemma 4 (see [35]). Consider two matrices $X$ and $\Phi$ with the compatible dimensions. The following two statements are equivalent.

(1) There exists a positive symmetric matrix $P$ such that

$$
X^{T} P X-\Phi<0
$$

(2) There exists a positive symmetric matrix $P$ and matrix $Z$ such that

$$
\left[\begin{array}{cc}
-\Phi & (*) \\
Z X & -Z-Z^{T}+P
\end{array}\right]<0
$$

\section{Decentralized Observer Design}

In this section, a polynomial observer is synthesized for system (3a) and (3b) to estimate the unknown states and eliminate the effects of the interconnection terms.

Let us consider the observer for system (3a) and (3b) as follows:

$$
\begin{gathered}
z_{l}(k+1)=\sum_{i=1}^{r_{l}} \beta_{i}^{l}\left(\theta^{l}(k)\right)\left\{N_{i}^{l}\left(\zeta_{l}(k)\right) z_{l}(k)\right. \\
\left.+G_{i}^{l}\left(\zeta_{l}(k)\right) u_{l}(k)+L_{i}^{l}\left(\zeta_{l}(k)\right) y_{l}(k)\right\} \\
\widehat{x}_{l}(k)=z_{l}(k)-J^{l} y_{l}(k)
\end{gathered}
$$

where $z_{l}(k)$ is the vector of the state variables of the observer (8a) and (8b). The polynomial matrices $N_{i}^{l}\left(\zeta_{l}(k)\right), G_{i}^{l}\left(\zeta_{l}(k)\right)$, $L_{i}^{l}\left(\zeta_{l}(k)\right)$ and constant matrix $J^{l}$ are the observer gains which will be determined in the next steps. It should be noted that, in this study, premise variables $\theta^{l}(k)$ are measured; hence, we can use these premise variables for the observer (8a) and (8b) and do not need to estimate them.

Let us define estimation errors of system (3a) and (3b) with observer $(8 \mathrm{a})$ and $(8 \mathrm{~b})$

$$
e_{l}(k)=\widehat{x}_{l}(k)-x_{l}(k)
$$

Substituting (3b) and ( $8 b)$ into (9) yields

$$
\begin{aligned}
e_{l}(k) & =z_{l}(k)-J^{l} y_{l}(k)-x_{l}(k) \\
& =z_{l}(k)-\left(I+J^{l} C^{l}\right) x_{l}(k)
\end{aligned}
$$

Denote

$$
H^{l}=\left(I+J^{l} C^{l}\right)
$$

then (9) is rewritten as follows:

$$
e_{l}(k)=z_{l}(k)-H^{l} x_{l}(k)
$$

The dynamic equation of estimation error is obtained

$$
e_{l}(k+1)=z_{l}(k+1)-H^{l} x_{l}(k+1)
$$

Combining (3a), (8a), and (13) yields

$$
\begin{aligned}
& e_{l}(k+1)=\sum_{i=1}^{r_{l}} \beta_{i}^{l}\left(\theta^{l}(k)\right)\left\{N_{i}^{l}\left(\zeta_{l}(k)\right) x_{l}(k)\right. \\
& \left.+G_{i}^{l}\left(\zeta_{l}(k)\right) u_{l}(k)+L_{i}^{l}\left(\zeta_{l}(k)\right) y_{l}(k)\right\} \\
& -H^{l} \sum_{i=1}^{r_{l}} \beta_{i}^{l}\left(\theta^{l}(k)\right)\left\{A_{i}^{l}\left(\zeta_{l}(k)\right) x_{l}(k)+B_{i}^{l}\left(\zeta_{l}(k)\right)\right. \\
& \left.\cdot u_{l}(k)+T^{l} \vartheta_{l}(x)\right\}=\sum_{i=1}^{r_{l}} \beta_{i}^{l}\left(\theta^{l}(k)\right)\left\{N_{i}^{l}\left(\zeta_{l}(k)\right) e_{l}(k)\right. \\
& +\left(N_{i}^{l}\left(\zeta_{l}(k)\right) H^{l}-H^{l} A_{i}^{l}\left(\zeta_{l}(k)\right)+L_{i}^{l}\left(\zeta_{l}(k)\right) C^{l}\right) \\
& \cdot x_{l}(k)+\left(G_{i}^{l}\left(\zeta_{l}(k)\right)-H^{l} B_{i}^{l}\left(\zeta_{l}(k)\right)\right) u_{l}(k) \\
& \left.+H^{l} T^{l} \vartheta_{l}(x)\right\}
\end{aligned}
$$

Theorem 5. Based on Assumption 1, the estimation error (9) of system (3a) and (3b) with observer (8a) and (8b) converges to zero asymptotically if there exist the polynomial matrices $N_{i}^{l}\left(\zeta_{l}(k)\right), G_{i}^{l}\left(\zeta_{l}(k)\right), L_{i}^{l}\left(\zeta_{l}(k)\right)$, constant matrix $J^{l}$, and symmetric matrices $P_{i}^{l}$ such that the following conditions hold:

$$
\begin{gathered}
N_{i}^{l}\left(\zeta_{l}(k)\right) H^{l}-H^{l} A_{i}^{l}\left(\zeta_{l}(k)\right)+L_{i}^{l}\left(\zeta_{l}(k)\right) C^{l}=0 \\
G_{i}^{l}\left(\zeta_{l}(k)\right)-H^{l} B_{i}^{l}\left(\zeta_{l}(k)\right)=0 \\
H^{l} T^{l}=0 \\
v_{1}^{T}\left(P_{i}^{l}-\varepsilon_{1 i}^{l} I\right) v_{1} \text { is SOS } \\
-v_{2}^{T}\left(\Xi_{i}^{l}\left(\zeta_{l}(k)\right)+\varepsilon_{2 i}^{l}\left(\zeta_{l}(k)\right) I\right) v_{2} \text { is SOS }
\end{gathered}
$$

where

$$
\begin{aligned}
\Xi_{i}^{l}\left(\zeta_{l}(k)\right) & =\left(N_{i}^{l}\left(\zeta_{l}(k)\right)\right)^{T} P_{m}^{l} N_{i}^{l}\left(\zeta_{l}(k)\right)-P_{i}^{l} \\
H^{l} & =\left(I+J^{l} C^{l}\right)
\end{aligned}
$$

$v_{1}$ and $v_{2}$ are the vectors which are independent on $\zeta_{l}(k), \varepsilon_{1 i}^{l}$ is a positive scalar, $\varepsilon_{2 i}^{l}\left(\zeta_{l}(k)\right)>0$ with $\zeta_{l}(k) \neq 0$.

Proof. If the conditions (15)-(17) hold, then (14) becomes

$$
e_{l}(k+1)=\sum_{i=1}^{r_{l}} \beta_{i}^{l}\left(\theta^{l}(k)\right)\left\{N_{i}^{l}\left(\zeta_{l}(k)\right) e_{l}(k)\right\}
$$

Select fuzzy Lyapunov function 


$$
V(k)=\sum_{l=1}^{N} V_{l}(k)=\sum_{l=1}^{N} \sum_{i=1}^{r_{l}} \beta_{i}^{l}\left(\theta^{l}(k)\right)\left\{e_{l}^{T}(k) P_{i}^{l} e_{l}(k)\right\}
$$

From condition (18) of Theorem 5, it implies that $P_{i}^{l}$ is a positive matrix. Thus, $V(k)>0$. From (22), we have

$$
\Delta V(k)=V(k+1)-V(k)
$$

Combining (22) and (23) obtains
$\Delta V(k)$

$$
\begin{aligned}
= & \sum_{l=1}^{N}\left\{\sum_{i=1}^{r_{l}} \beta_{i}^{l}\left(\theta^{l}(k+1)\right)\left[e_{l}^{T}(k+1) P_{i}^{l} e_{l}(k+1)\right]\right. \\
- & \left.\sum_{i=1}^{r_{l}} \beta_{i}^{l}\left(\theta^{l}(k)\right)\left[e_{l}^{T}(k) P_{i}^{l} e_{l}(k)\right]\right\}
\end{aligned}
$$

Substituting (21) into (24) yields

$$
\Delta V(k)=\sum_{l=1}^{N}\left\{\sum_{i=1}^{r_{l}} \sum_{j=1}^{r_{l}} \sum_{m=1}^{r_{l}} \beta_{i}^{l}\left(\theta^{l}(k)\right) \beta_{j}^{l}\left(\theta^{l}(k)\right) \beta_{m}^{l}\left(\theta^{l}(k+1)\right) e_{l}^{T}(k)\left[\left(N_{j}^{l}\left(\zeta_{l}(k)\right)\right)^{T} P_{m}^{l} N_{i}^{l}\left(\zeta_{l}(k)\right)-P_{i}^{l}\right] e_{l}(k)\right\}
$$

Define

$$
\begin{gathered}
\Phi_{m}^{l}\left(\zeta_{l}(k)\right)=\sum_{i=1}^{r_{l}} \sum_{j=1}^{r_{l}} \beta_{i}^{l}\left(\theta^{l}(k)\right) \beta_{j}^{l}\left(\theta^{l}(k)\right) e_{l}^{T}(k) \\
\cdot\left[\left(N_{j}^{l}\left(\zeta_{l}(k)\right)\right)^{T} P_{m}^{l} N_{i}^{l}\left(\zeta_{l}(k)\right)-P_{i}^{l}\right] e_{l}(k)
\end{gathered}
$$

From (25) and (26), one obtains

$$
\Delta V(k)=\sum_{l=1}^{N}\left\{\sum_{m=1}^{r_{l}} \beta_{m}^{l}\left(\theta^{l}(k+1)\right) \Phi_{m}^{l}\left(\zeta_{l}(k)\right)\right.
$$

From (26), it infers that

$$
\begin{aligned}
\Phi_{m}^{l}\left(\zeta_{l}(k)\right) & =\sum_{i=1}^{n_{1}}\left[\beta_{i}^{l}\left(\theta^{l}(k)\right)\right]^{2} \\
& \cdot e_{l}^{T}(k)\left\{\left(N_{i}^{l}\left(\zeta_{l}(k)\right)\right)^{T} P_{m}^{l} N_{i}^{l}\left(\zeta_{l}(k)\right)-P_{i}^{l}\right\} e_{l}(k) \\
& +\sum_{i=1}^{r_{l}} \sum_{j=1}^{n_{i}} \beta_{i}^{l}\left(\theta^{l}(k)\right) \beta_{j}^{l}\left(\theta^{l}(k)\right) e_{l}^{T}(k) \\
& \cdot\left\{\left(N_{i}^{l}\left(\zeta_{l}(k)\right)\right)^{T} P_{m}^{l} N_{j}^{l}\left(\zeta_{l}(k)\right)-P_{i}^{l}+\left(N_{j}^{l}\left(\zeta_{l}(k)\right)\right)^{T} P_{m}^{l} N_{i}^{l}\left(\zeta_{l}(k)\right)-P_{j}^{l}\right\} e_{l}(k)
\end{aligned}
$$

Applying Lemma 3 for (28) obtains

$$
\begin{aligned}
\Phi_{m}^{l}\left(\zeta_{l}(k)\right) & \leq \sum_{i=1}^{n_{i}}\left[\beta_{i}^{l}\left(\theta^{l}(k)\right)\right]^{2} \\
& \cdot e_{l}^{T}(k)\left\{\left(N_{i}^{l}\left(\zeta_{l}(k)\right)\right)^{T} P_{m}^{l} N_{i}^{l}\left(\zeta_{l}(k)\right)-P_{i}^{l}\right\} e_{l}(k) \\
& +\sum_{i=1}^{r_{l}} \sum_{j=1}^{r_{i}} \beta_{i}^{l}\left(\theta^{l}(k)\right) \beta_{j}^{l}\left(\theta^{l}(k)\right) e_{l}^{T}(k) \\
& \cdot\left\{\left(N_{i}^{l}\left(\zeta_{l}(k)\right)\right)^{T} P_{m}^{l} N_{i}^{l}\left(\zeta_{l}(k)\right)-P_{i}^{l}+\left(N_{j}^{l}\left(\zeta_{l}(k)\right)\right)^{T} P_{m}^{l} N_{j}^{l}\left(\zeta_{l}(k)\right)-P_{j}^{l}\right\} e_{l}(k)
\end{aligned}
$$

It is easily seen that

$$
\begin{aligned}
& \sum_{i=1}^{r_{l}}\left[\beta_{i}^{l}\left(\theta^{l}(k)\right)\right]^{2} e_{l}^{T}(k)\left\{\left(N_{i}^{l}\left(\zeta_{l}(k)\right)\right)^{T} P_{m}^{l} N_{i}^{l}\left(\zeta_{l}(k)\right)-P_{i}^{l}\right\} e_{l}(k) \\
& +\sum_{i=1}^{r_{l}} \sum_{j=1}^{r_{l}} \beta_{i}^{l}\left(\theta^{l}(k)\right) \beta_{j}^{l}\left(\theta^{l}(k)\right) e_{l}^{T}(k) \\
& \cdot\left\{\left(N_{i}^{l}\left(\zeta_{l}(k)\right)\right)^{T} P_{m}^{l} N_{i}^{l}\left(\zeta_{l}(k)\right)-P_{i}^{l}+\left(N_{j}^{l}\left(\zeta_{l}(k)\right)\right)^{T} P_{m}^{l} N_{j}^{l}\left(\zeta_{l}(k)\right)-P_{j}^{l}\right\} e_{l}(k) \\
& =\sum_{i=1}^{n_{n}} \beta_{i}^{l}\left(\theta^{l}(k)\right) e_{l}^{T}(k)\left\{\left(N_{i}^{l}\left(\zeta_{l}(k)\right)\right)^{T} P_{m}^{l} N_{i}^{l}\left(\zeta_{l}(k)\right)-P_{i}^{l}\right\} e_{l}(k)
\end{aligned}
$$

therefore,

$$
\begin{aligned}
\Phi_{m}^{l}\left(\zeta_{l}(k)\right) \leq & \sum_{i=1}^{r_{l}} \beta_{i}^{l}\left(\theta^{l}(k)\right) e_{l}^{T}(k) \\
& \cdot\left\{\left(N_{i}^{l}\left(\zeta_{l}(k)\right)\right)^{T} P_{m}^{l} N_{i}^{l}\left(\zeta_{l}(k)\right)-P_{i}^{l}\right\} \\
& \cdot e_{l}(k)
\end{aligned}
$$

From (31), it leads to

$$
\begin{aligned}
& \Delta V(k)=\sum_{l=1}^{N}\left\{\sum_{i=1}^{r_{l}} \beta_{i}^{l}\left(\theta^{l}(k+1)\right) \Phi_{m}^{l}\left(\zeta_{l}(k)\right)\right\} \\
& \leq \sum_{i=1}^{r_{l}} \beta_{i}^{l}\left(\theta^{l}(k)\right) e_{l}^{T}(k) \\
& \cdot\left\{\left(N_{i}^{l}\left(\zeta_{l}(k)\right)\right)^{T} P_{m}^{l} N_{i}^{l}\left(\zeta_{l}(k)\right)-P_{i}^{l}\right\} e_{l}(k)
\end{aligned}
$$

It is obvious that if condition (19) holds, then $\Delta V(k)<$ 0 . It means that the estimation errors (9) approach zero asymptotically. The proof is completed.

However, condition (19) is a polynomial Bilinear Matrix Inequality which makes it very difficult to obtain the results in Matlab by using SOS tool. Thus, a transformation to convert (19) into the Polynomial Linear Matrix Inequality is needed.

Theorem 6. Based on Assumption 1, the estimated state variables of system (3a) and (3b) with the observer (8a) and (8b) approach the real states asymptotically if there exist the polynomial matrices $N_{i}^{l}\left(\zeta_{l}(k)\right), G_{i}^{l}\left(\zeta_{l}(k)\right), L_{i}^{l}\left(\zeta_{l}(k)\right)$, $Z^{l}\left(\zeta_{l}(k)\right), J^{l}$, and symmetric matrices $P_{i}^{l}$ such that the following conditions satisfy

$v_{1}^{T}\left(P_{i}^{l}-\varepsilon_{1 i}^{l}\right) v_{1}$ is SOS

$-v_{2}^{T}\left\{\left[\begin{array}{cc}-P_{m}^{l} & (*) \\ \Omega_{i}^{l}\left(\zeta_{l}(k)\right) & -Z^{l}\left(\zeta_{l}(k)\right)-\left[Z^{l}\left(\zeta_{l}(k)\right)\right]^{T}+P_{m}^{l}\end{array}\right]+\varepsilon_{2 i}^{l}\left(\zeta_{l}(k)\right) I\right\} v_{2}$ is SOS 
in which

$$
\begin{aligned}
& \Omega_{i}^{l}\left(\zeta_{l}(k)\right)=Z^{l}\left(\zeta_{l}(k)\right)\left(I+U^{l} C^{l}\right) A_{i}^{l}\left(\zeta_{l}(k)\right) \\
& \quad+Q^{l}\left(\zeta_{l}(k)\right) V^{l} C^{l} A_{i}^{l}\left(\zeta_{l}(k)\right)-M_{i}^{l}\left(\zeta_{l}(k)\right) C^{l} \\
& Q^{l}\left(\zeta_{l}(k)\right)=Z^{l}\left(\zeta_{l}(k)\right) Y^{l} \\
& M_{i}^{l}\left(\zeta_{l}(k)\right)=Z^{l}\left(\zeta_{l}(k)\right) K_{i}^{l}\left(\zeta_{l}(k)\right) \\
& U^{l}=-T^{l}\left(C^{l} T^{l}\right)^{+} \\
& V^{l}=I-\left(C^{l} T^{l}\right)\left(C^{l} T^{l}\right)^{+} \\
& H^{l}=I+J^{l} C^{l}
\end{aligned}
$$

Observer gains are obtained as follows:

$$
\begin{aligned}
J^{l} & =U^{l}+Y^{l} V^{l} \\
G_{i}^{l}\left(\zeta_{l}(k)\right) & =H^{l} B_{i}^{l}\left(\zeta_{l}(k)\right) \\
L_{i}^{l}\left(\zeta_{l}(k)\right) & =K_{i}^{l}\left(\zeta_{l}(k)\right)\left(I+C^{l} J^{l}\right)-H^{l} A_{i}^{l}\left(\zeta_{l}(k)\right) J^{l} \\
N_{i}^{l}\left(\zeta_{l}(k)\right) & =H^{l} A_{i}^{l}\left(\zeta_{l}(k)\right)-K_{i}^{l}\left(\zeta_{l}(k)\right) C^{l}
\end{aligned}
$$

$\left(C^{l} T^{l}\right)^{+}$is the Moore-Penrose pseudoinverse of $\left(C^{l} T^{l}\right), i=$ $1,2, \ldots, r_{l}, l=1,2, \ldots, N$.

Proof. From condition (19) of Theorem 5, it infers that

$$
\Xi_{i}^{l}\left(\zeta_{l}(k)\right)=\left(N_{i}^{l}\left(\zeta_{l}(k)\right)\right)^{T} P_{m}^{l} N_{i}^{l}\left(\zeta_{l}(k)\right)-P_{i}^{l}<0
$$

Employing Lemma 4 for condition (45) of Theorem 5 with a slack variable $Z^{l}\left(\zeta_{l}(k)\right)$ yields

$$
\begin{aligned}
& {\left[\begin{array}{cc}
-P_{m}^{l} & (*) \\
Z^{l}\left(\zeta_{l}(k)\right) N_{i}^{l}\left(\zeta_{l}(k)\right) & -Z^{l}\left(\zeta_{l}(k)\right)-\left[Z^{l}\left(\zeta_{l}(k)\right)\right]^{T}+P_{m}^{l}
\end{array}\right]} \\
& <0
\end{aligned}
$$

From condition (17), we have

$$
J^{l}\left(C^{l} T^{l}\right)=-T^{l}
$$

The general solution of (47) is

$$
J^{l}=U^{l}+Y^{l} V^{l}
$$

where

$$
\begin{aligned}
& U^{l}=-T^{l}\left(C^{l} T^{l}\right)^{+} \\
& V^{l}=I-\left(C^{l} T^{l}\right)\left(C^{l} T^{l}\right)^{+}
\end{aligned}
$$

$\left(C^{l} T^{l}\right)^{+}=\left(\left(C^{l} T^{l}\right)^{T}\left(C^{l} T^{l}\right)\right)^{-1}\left(C^{l} T^{l}\right)^{T}$ is the MoorePenrose pseudoinverse of $\left(C^{l} T^{l}\right)$

$Y^{l}$ is an arbitrary matrix with the compatible dimension.

Define

$$
K_{i}^{l}\left(\zeta_{l}(k)\right)=L_{i}^{l}\left(\zeta_{l}(k)\right)+N_{i}^{l}\left(\zeta_{l}(k)\right) J^{l}
$$

Combining (15) and (49) yields

$$
\begin{aligned}
& N_{i}^{l}\left(\zeta_{l}(k)\right)=H^{l} A_{i}^{l}\left(\zeta_{l}(k)\right)-K_{i}^{l}\left(\zeta_{l}(k)\right) C^{l} \\
& L_{i}^{l}\left(\zeta_{l}(k)\right)=K_{i}^{l}\left(\zeta_{l}(k)\right)\left(I+C^{l} J^{l}\right)-H^{l} A_{i}^{l}\left(\zeta_{l}(k)\right) J^{l}
\end{aligned}
$$

From (48) and (50), one obtains

$$
\begin{aligned}
N_{i}^{l}\left(\zeta_{l}(k)\right)= & \left(I+\left(U^{l}+Y^{l} V^{l}\right) C^{l}\right) A_{i}^{l}\left(\zeta_{l}(k)\right) \\
& -K_{i}^{l}\left(\zeta_{l}(k)\right) C^{l}
\end{aligned}
$$

Substituting (52) into (46) yields

$$
\left[\begin{array}{c}
-P_{i}^{l} \\
Z^{l}\left(\zeta_{l}(k)\right)\left[\left(I+\left(U^{l}+Y^{l} V^{l}\right) C^{l}\right) A_{i}^{l}\left(\zeta_{l}(k)\right)-K_{i}^{l}\left(\zeta_{l}(k)\right) C^{l}\right] \\
-Z^{l}\left(\zeta_{l}(k)\right)-\left[Z^{l}\left(\zeta_{l}(k)\right)\right]^{T}+P_{i}^{l}
\end{array}\right]<0
$$

Let us denote

$$
\begin{aligned}
Q^{l}\left(\zeta_{l}(k)\right) & =Z^{l}\left(\zeta_{l}(k)\right) Y^{l} \\
M_{i}^{l}\left(\zeta_{l}(k)\right) & =Z^{l}\left(\zeta_{l}(k)\right) K_{i}^{l}\left(\zeta_{l}(k)\right)
\end{aligned}
$$

From (53), (54), and (55), we obtain

$$
\left[\begin{array}{cc}
-P_{i}^{l} & (*) \\
\Omega_{i}^{l}\left(\zeta_{l}(k)\right) & -Z^{l}\left(\zeta_{l}(k)\right)-\left[Z^{l}\left(\zeta_{l}(k)\right)\right]^{T}+P_{i}^{l}
\end{array}\right]<0
$$

where

$$
\begin{aligned}
\Omega_{i}^{l}\left(\zeta_{l}(k)\right)= & Z^{l}\left(\zeta_{l}(k)\right)\left(I+U^{l} C^{l}\right) A_{i}^{l}\left(\zeta_{l}(k)\right) \\
& +Q^{l}\left(\zeta_{l}(k)\right) V^{l} C^{l} A_{i}^{l}\left(\zeta_{l}(k)\right) \\
& -M_{i}^{l}\left(\zeta_{l}(k)\right) C^{l}
\end{aligned}
$$

From (55), it is seen that condition (19) of Theorem 5 has been transformed into PLMI (34) of Theorem 6 which can be solved easily in Matlab. Hence, the proof is completed. 
For the sake of understanding easily, the procedure for observer design is briefed as follows.

Step 1. Compute $U^{l}$ and $V^{l}$ from (38) and (39).

Step 2. Substitute $A_{i}^{l}\left(\zeta_{l}(k)\right), U^{l}, V^{l}$, and $C^{l}$ into (34), then solving this SOS by using SOS tool of Matlab obtains $P_{m}^{l}$, $Z^{l}\left(\zeta_{l}(k)\right), Q^{l}\left(\zeta_{l}(k)\right)$, and $M_{i}^{l}\left(\zeta_{l}(k)\right)$.

Step 3. Calculate $Y^{l}$ and $K_{i}^{l}\left(\zeta_{l}(k)\right)$ from (36) and (37), respectively. After that, $H^{l}$ is determined by (40).

Step 4. The observer gains $J^{l}, G_{i}^{l}\left(\zeta_{l}(k)\right), \quad L_{i}^{l}\left(\zeta_{l}(k)\right)$, and $N_{i}^{l}\left(\zeta_{l}(k)\right)$, are computed from (41)-(44).

Step 5. With observer gains in Step 4, observer (8a) and (8b) is constructed.

Remark 7. In $[22,25]$, the interconnection terms are required to satisfy the bounded constraint; however, the considered interconnection parts in this work are arbitrary and do not need to satisfy any bounded constraint; therefore, the methods in $[22,25]$ failed to design the observer for these systems while our method is still applicable. In addition, the method in [21, 26-29] is applicable when the interconnection terms can be linearized under the linear framework or T-S fuzzy model. However, in practice, in some systems, it is very difficult to transform the interconnection terms to the linear or T-S fuzzy model forms; it cause the method in [21, 2629] to be unable to apply for these systems. Even if these interconnection parts can be linearized under the linear form or T-S fuzzy model as in [21, 26-29], it also has the following drawbacks: (1) the modeling error between the original largescale nonlinear system and the modeled system will increase and (2) the number of the fuzzy rules, the complexity, and computational load significantly grows up.

Remark 8. Regarding the equality conditions (15)-(17) of Theorem 5, they have been integrated to transform the PBMI (Polynomial Bilinear Matrix Inequality) (19) into the PLMI (Polynomial Linear Matrix Inequality) (34) of Theorem 6. Then by using SOS tool of Matlab, it is easy to find the feasible solution of PLMI (34) and these obtained results are guaranteed satisfying the equality conditions (15)-(17) as well.

\section{Illustrative Example}

Take into account a discrete-time nonlinear large-scale system with three interconnection subsystems as follows:

Subsystem 1

$$
\begin{aligned}
x_{11}(k+1)= & -0.1 x_{11}(k) \\
& +0.1 x_{11}(k) x_{21}(k) \cos \left(x_{11}(k)\right) \\
& -0.2 x_{21}(k) \cos \left(x_{22}(k)\right) \\
& -0.2 x_{13}(k) \sin \left(x_{23}(k)\right)+u_{1}(k)
\end{aligned}
$$

$$
\begin{aligned}
x_{21}(k+1)= & 0.1 x_{11}(k)-0.1 x_{11}^{2}(k) x_{21}(k) \\
& -x_{21}(k) \cos \left(x_{22}(k)\right) \\
& -x_{13}(k) \sin \left(x_{23}(k)\right)+u_{1}(k) \\
y_{1}(k)= & x_{11}(k)
\end{aligned}
$$

Subsystem 2

$$
\begin{aligned}
x_{12}(k+1)= & -0.1 x_{12}(k)-0.1 x_{12}^{2}(k) x_{22}(k) \\
& +0.5 x_{11}(k) \times \sin \left(x_{12}(k)\right) \\
& +0.5 x_{23}(k) \cos \left(x_{13}(k)\right)+u_{2}(k) \\
x_{22}(k+1)= & -0.1 x_{12}(k) \cos \left(x_{12}(k)\right) \\
& -0.5 x_{22}(k) 2 x_{11}(k) \sin \left(x_{12}(k)\right) \\
& \left.+2 x_{23}(k) \cos \left(x_{13}(k)\right)\right)+u_{2}(k) \\
y_{2}(k)= & x_{12}(k)
\end{aligned}
$$

Subsystem 3

$$
\begin{aligned}
x_{13}(k+1)= & -0.2 x_{13}(k) \\
& +0.1\left(x_{13}(k)+1\right) x_{23}(k) \cos \left(x_{13}(k)\right) \\
& +0.2 x_{11}(k) \sin \left(x_{21}(k)\right) \\
& -0.2 x_{22}(k) \cos \left(x_{12}(k)\right)+u_{3}(k) \\
x_{23}(k+1)= & -0.5 x_{13}(k) \cos \left(x_{13}(k)\right) \\
& -0.1 x_{13}^{2}(k) x_{23}(k) \\
& +x_{11}(k) \sin \left(x_{21}(k)\right) \\
& -x_{22}(k) \cos \left(x_{12}(k)\right)+u_{3}(k) \\
y_{3}(k)= & x_{13}(k)
\end{aligned}
$$

The interconnection terms:

$$
\begin{aligned}
& g_{1}(x) \\
& =\left[\begin{array}{c}
-0.2 x_{21}(k) \cos \left(x_{22}(k)\right)-0.2 x_{13}(k) \sin \left(x_{23}(k)\right) \\
-x_{21}(k) \cos \left(x_{22}(k)\right)-x_{13}(k) \sin \left(x_{23}(k)\right)
\end{array}\right] \\
& g_{2}(x) \\
& =\left[\begin{array}{c}
0.5 x_{11}(k) \sin \left(x_{12}(k)\right)+0.5 x_{23}(k) \cos \left(x_{13}(k)\right) \\
\left.2 x_{11}(k) \sin \left(x_{12}(k)\right)+2 x_{23}(k) \cos \left(x_{13}(k)\right)\right)
\end{array}\right] \\
& g_{3}(x) \quad\left[\begin{array}{c}
0.2 x_{11}(k) \sin \left(x_{21}(k)\right)-0.2 x_{22}(k) \cos \left(x_{12}(k)\right) \\
x_{11}(k) \sin \left(x_{21}(k)\right)-x_{22}(k) \cos \left(x_{12}(k)\right)
\end{array}\right]
\end{aligned}
$$


In this example, rewriting system (58a), (58b), and (58c) under the framework of system (3a) and (3b) with $\zeta_{l}(k)=$ $y_{l}(k)$ yields

$$
\begin{aligned}
& x_{l}(k+1)=\sum_{i=1}^{r_{l}} \beta_{i}^{l}\left(\theta^{l}(k)\right) \\
& \cdot\left\{A_{i}^{l}\left(y_{l}\right) x_{l}(k)+B_{i}^{l}\left(y_{l}\right) u_{l}(k)+T^{l} \vartheta_{l}(x)\right. \\
& y_{l}(k)=C^{l} x_{l}(k), \quad l=1,2,3
\end{aligned}
$$

where

$$
\begin{aligned}
x_{1}(k) & =\left[\begin{array}{l}
x_{11}(k) \\
x_{21}(k)
\end{array}\right] ; x_{2}(k)=\left[\begin{array}{l}
x_{12}(k) \\
x_{22}(k)
\end{array}\right] ; x_{3}(k) \\
& =\left[\begin{array}{l}
x_{13}(k) \\
x_{23}(k)
\end{array}\right] ; \\
A_{1}^{1}\left(y_{1}\right) & =\left[\begin{array}{cc}
-0.1 & 0.1 y_{1} \\
0.1 & -0.1 y_{1}^{2}
\end{array}\right], \\
B_{1}^{1}\left(y_{1}\right) & =B_{2}^{1}\left(y_{1}\right)=\left[\begin{array}{l}
1 \\
1
\end{array}\right] \\
A_{2}^{1}\left(y_{1}\right) & =\left[\begin{array}{cc}
-0.1 & -0.1 y_{1} \\
0.1 & -0.1 y_{1}^{2}
\end{array}\right], \\
C^{1} & =\left[\begin{array}{lll}
1 & 0
\end{array}\right], \quad T^{1}=\left[\begin{array}{l}
0.2 \\
1
\end{array}\right] .
\end{aligned}
$$

$$
\begin{aligned}
\vartheta_{1}(x)= & -x_{21}(k) \cos \left(x_{22}(k)\right) \\
& -x_{13}(k) \sin \left(x_{23}(k)\right)
\end{aligned}
$$$$
A_{1}^{2}\left(y_{2}\right)=\left[\begin{array}{cc}
-0.1 & -0.1 y_{2}^{2} \\
-0.1 & -0.5
\end{array}\right] \text {, }
$$$$
A_{2}^{2}\left(y_{2}\right)=\left[\begin{array}{cc}
-0.1 & -0.1 y_{2}^{2} \\
0.1 & -0.5
\end{array}\right] \text {, }
$$$$
B_{1}^{2}\left(y_{2}\right)=B_{2}^{2}\left(y_{2}\right)=\left[\begin{array}{l}
1 \\
1
\end{array}\right]
$$

$$
C^{2}=\left[\begin{array}{ll}
1 & 0
\end{array}\right], \quad T^{2}=\left[\begin{array}{c}
0.5 \\
2
\end{array}\right] .
$$$$
\left.\vartheta_{2}(x)=x_{11}(k) \sin \left(x_{12}(k)\right)+x_{23}(k) \cos \left(x_{13}(k)\right)\right)
$$$$
A_{1}^{3}\left(y_{3}\right)=\left[\begin{array}{cc}
-0.2 & 0.1 y_{3}+0.1 \\
-0.5 & -0.1 y_{3}^{2}
\end{array}\right] \text {, }
$$$$
A_{2}^{3}\left(y_{3}\right)=\left[\begin{array}{cc}
-0.2 & -0.1 y_{3}-0.1 \\
0.5 & -0.1 y_{3}^{2}
\end{array}\right] \text {, }
$$$$
B_{1}^{3}\left(y_{3}\right)=B_{2}^{3}\left(y_{3}\right)=\left[\begin{array}{l}
1 \\
1
\end{array}\right]
$$$$
C^{3}=\left[\begin{array}{ll}
1 & 0
\end{array}\right], \quad T^{3}=\left[\begin{array}{c}
0.2 \\
1
\end{array}\right] .
$$$$
\vartheta_{3}(x)=x_{11}(k) \sin \left(x_{21}(k)\right)-x_{22}(k) \cos \left(x_{12}(k)\right)
$$

Applying the method in Section 3 and solving the conditions of Theorems 5 and 6 yields

$$
\begin{aligned}
& N_{1}^{1}\left(y_{1}\right)=\left[\begin{array}{cc}
-0.229 * 10^{-15} y_{1}^{2}+0.298 * 10^{-12} y_{1}-0.815 * 10^{-7} & 0 \\
0.004 y_{1}^{2}+95.38 y_{1}+2.86 & -0.1 y_{1}^{2}-0.5 y_{1}
\end{array}\right] \\
& N_{2}^{1}\left(y_{1}\right)=\left[\begin{array}{cc}
-0.225 * 10^{-15} y_{1}^{2}+0.308 * 10^{-12} y_{1}-0.815 * 10^{-7} & 0 \\
0.004 y_{1}^{2}+95.36 y_{1}+2.59 & -0.1 y_{1}^{2}+0.5 y_{1}
\end{array}\right] \\
& N_{1}^{2}\left(y_{2}\right)=\left[\begin{array}{cc}
0.10 * 10^{-16} y_{2}^{2}+0.55 * 10^{-7} y_{2}+0.154 * 10^{-3} & 0 \\
0.769 * 10^{-10} y_{2}^{2}-0.55 * 10^{-7} y_{2}+165.31 & 0.4 y_{2}^{2}-0.5
\end{array}\right] \\
& N_{2}^{2}\left(y_{2}\right)=\left[\begin{array}{cc}
0.30 * 10^{-17} y_{2}^{2}+0.105 * 10^{-15} y_{2}+0.133 * 10^{-3} & 0 \\
-0.934 * 10^{-9} y_{2}^{2}-0.124 * 10^{-6} y_{2}+213.0 & 0.4 y_{2}^{2}-0.5
\end{array}\right] \\
& N_{1}^{3}\left(y_{2}\right)=\left[\begin{array}{cc}
-0.91 * 10^{-14} y_{3}^{2}+0.619 * 10^{-8} y_{3}+0.127 * 10^{-2} & 0 \\
0.0025 y_{3}^{2}-43.27 y_{3}-302.95 & 0.2 y_{3}^{2}-0.5 y_{3}-0.5
\end{array}\right] \\
& N_{2}^{3}\left(y_{2}\right)=\left[\begin{array}{cc}
-0.406 * 10^{-13} y_{3}^{2}+0.904 * 10^{-8} y_{3}+0.263 * 10^{-2} & 0 \\
0.007 y_{3}^{2}+31.17 y_{3}-455.25 & -0.2 y_{3}^{2}+0.5 y_{3}+0.5
\end{array}\right]
\end{aligned}
$$




$$
\begin{aligned}
& J^{1}=\left[\begin{array}{l}
-1 \\
-5
\end{array}\right], J^{2}=\left[\begin{array}{l}
-1 \\
-4
\end{array}\right], J^{3}=\left[\begin{array}{l}
-1 \\
-5
\end{array}\right] \text {, } \\
& G_{1}^{1}=\left[\begin{array}{c}
0 \\
-4
\end{array}\right], G_{2}^{1}=\left[\begin{array}{c}
0 \\
-4
\end{array}\right], G_{1}^{2}=\left[\begin{array}{c}
0 \\
-3
\end{array}\right] \text {, } \\
& G_{2}^{2}=\left[\begin{array}{c}
0 \\
-3
\end{array}\right], G_{1}^{3}=\left[\begin{array}{c}
0 \\
-4
\end{array}\right], G_{2}^{3}=\left[\begin{array}{c}
0 \\
-4
\end{array}\right] \\
& L_{1}^{1}\left(y_{1}\right)=\left[\begin{array}{c}
0 \\
-0.5 y_{1}^{2}-2.5 y_{1}+0.6
\end{array}\right] \text {, } \\
& L_{2}^{1}\left(y_{1}\right)=\left[\begin{array}{c}
0 \\
-0.5 y_{1}^{2}+2.5 y_{1}+0.6
\end{array}\right] \\
& L_{1}^{2}\left(y_{2}\right)=\left[\begin{array}{c}
0 \\
1.6 y_{2}^{2}-0.17
\end{array}\right] \text {, } \\
& L_{2}^{2}\left(y_{2}\right)=\left[\begin{array}{c}
0 \\
1.6 y_{2}^{2}-1.5
\end{array}\right] \\
& L_{1}^{3}\left(y_{3}\right)=\left[\begin{array}{c}
0 \\
-0.5 y_{3}^{2}-2.5 y_{3}-2
\end{array}\right] \text {, } \\
& L_{2}^{3}\left(y_{3}\right)=\left[\begin{array}{c}
0 \\
-0.5 y_{3}^{2}+2.5 y_{3}+4
\end{array}\right]
\end{aligned}
$$

Simulating the system in Matlab, we obtain the results in Figures 1-6.

The real states $x_{11}(k), x_{21}(k)$, estimated states $\widehat{x}_{11}(k)$, $\widehat{x}_{21}(k)$, and estimation errors $e_{11}(k), e_{21}(k)$ of the subsystem 1 are shown in Figures 1 and 2; Figures 3 and 4 illustrated the real states $x_{12}(k), x_{22}(k)$, estimated states $\widehat{x}_{12}(k), \widehat{x}_{22}(k)$, and estimation errors $e_{12}(k), e_{22}(k)$ of the subsystem 2 . Finally, the real states $x_{13}(k), x_{23}(k)$, estimated states $\widehat{x}_{13}(k), \widehat{x}_{23}(k)$, and estimation errors $e_{13}(k), e_{23}(k)$ are demonstrated in Figures 5 and 6 , respectively. From these simulation results, it is obvious that all estimated states approach the real states and the estimation errors converge to zero asymptotically. Hence, we can conclude that the proposed method in this paper is successful to design an observer for the large-scale system (58a), (58b), and (58c).

Remark 9. It should be noted that if we employ the T-S fuzzy model to represent system (58a), (58b), and (58c), then the number of fuzzy rules in each subsystem will double making the number of the conditions for observer design exponentially grows up. Moreover, it is seen that we do not know the upper bounds of the interconnection terms $g_{1}(x)$, $g_{2}(x), g_{3}(x)$ in this example; thus, the methods in $[22,25]$ are unable to apply for this system. In addition, because the interconnection terms $g_{l}(x), l=1,2,3$ of system (58a), (58b), and $(58 \mathrm{c})$ in this example contain the nonlinear function of states from other interconnection systems, it is not possible to model system (58a), (58b), and (58c) under the framework of the T-S fuzzy model as [8-15, 21, 26-29]. Hence, the methods in $[8-15,21,26-29]$ failed to apply to design observer for system (58a), (58b), and (58c).

\section{Conclusions}

A new approach based on the unknown input method to synthesize an observer for the discrete-time large-scale polynomial nonlinear system is studied in this paper. The discretetime large-scale nonlinear system is modeled in terms of a group of discrete-time polynomial T-S fuzzy subsystems to decrease the number of fuzzy rules. The interconnection parts in this system are arbitrary and they do not require the bounded constraints. By employing the unknown input method, the influences of the interconnection terms are eliminated completely and the unmeasurable state variables of the system are estimated asymptotically. The conditions represented under the form of SOS for synthesizing observer are derived in the two theorems. An illustrative example is presented to demonstrate the success and effectiveness of the proposed method.

\section{Data Availability}

No data were used to support this study. 


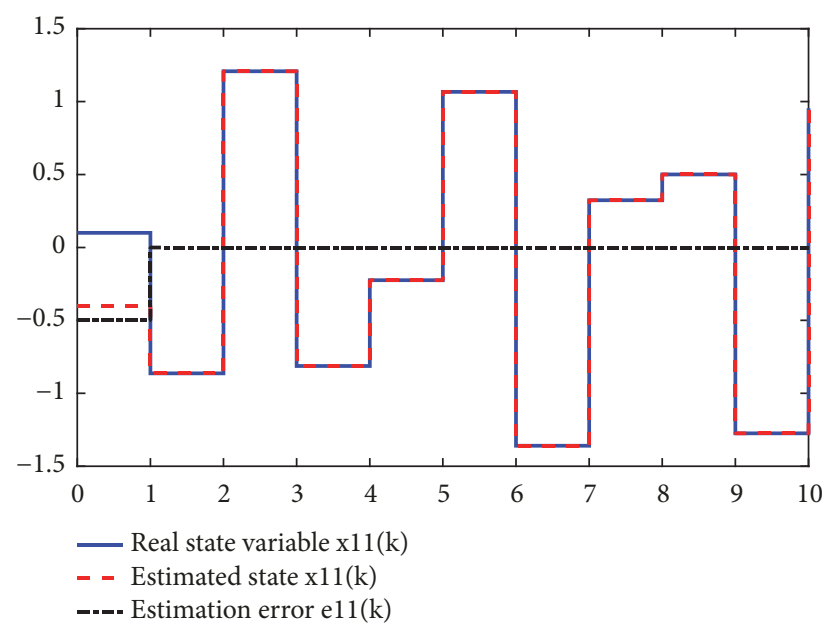

FIGURE 1: Real state $x_{11}(k)$, estimated state $\widehat{x}_{11}(k)$, and estimation error $e_{11}(k)$.

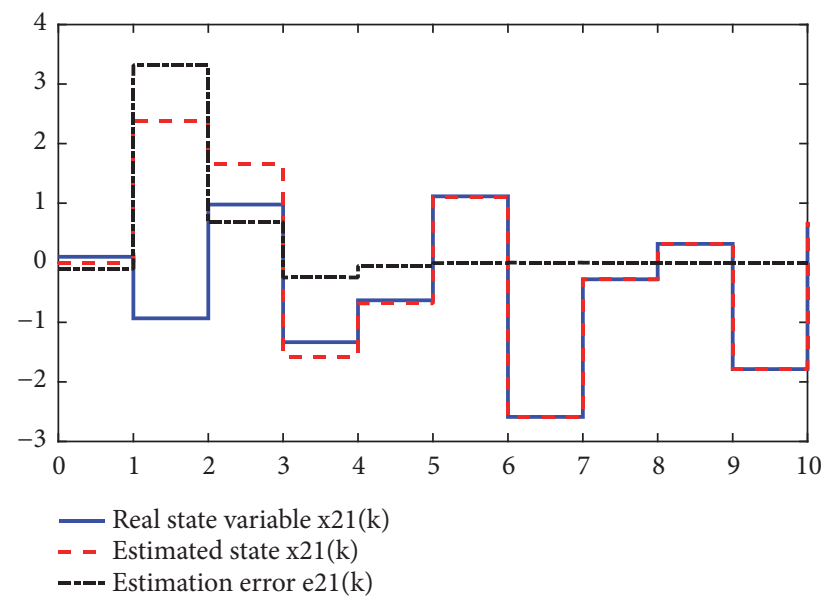

FIGURE 2: Real state $x_{21}(k)$, estimated state $\hat{x}_{21}(k)$, and estimation error $e_{21}(k)$.

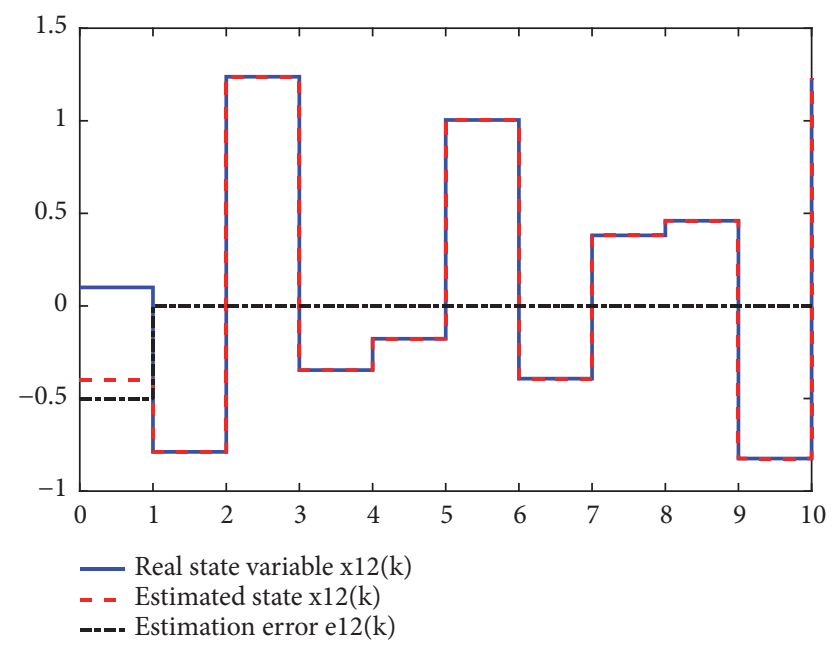

FIGURE 3: Real state $x_{12}(k)$, estimated state $\widehat{x}_{12}(k)$, and estimation error $e_{12}(k)$.

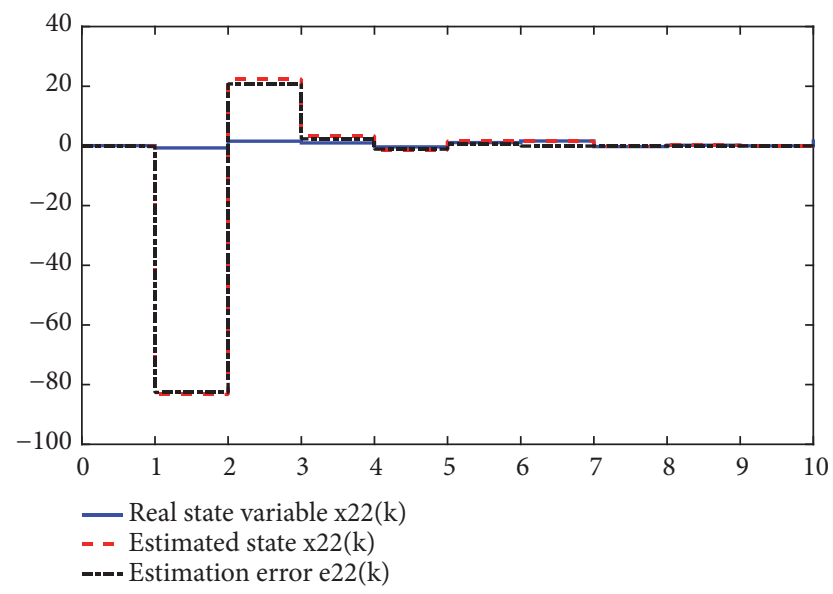

FIGURE 4: Real state $x_{22}(k)$, estimated state $\widehat{x}_{22}(k)$, and estimation error $e_{22}(k)$.

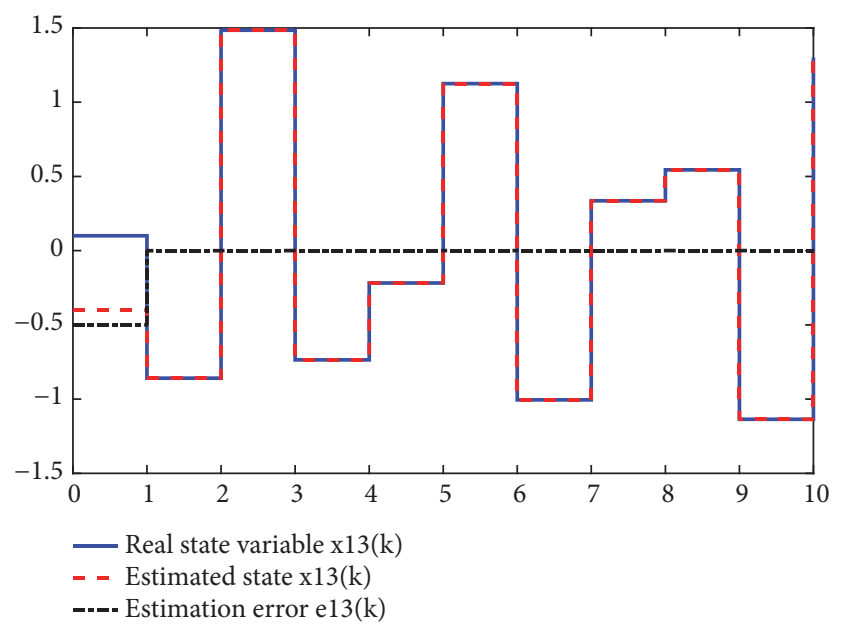

FIGURE 5: Real state $x_{13}(k)$, estimated state $\widehat{x}_{13}(k)$, and estimation error $e_{13}(k)$.

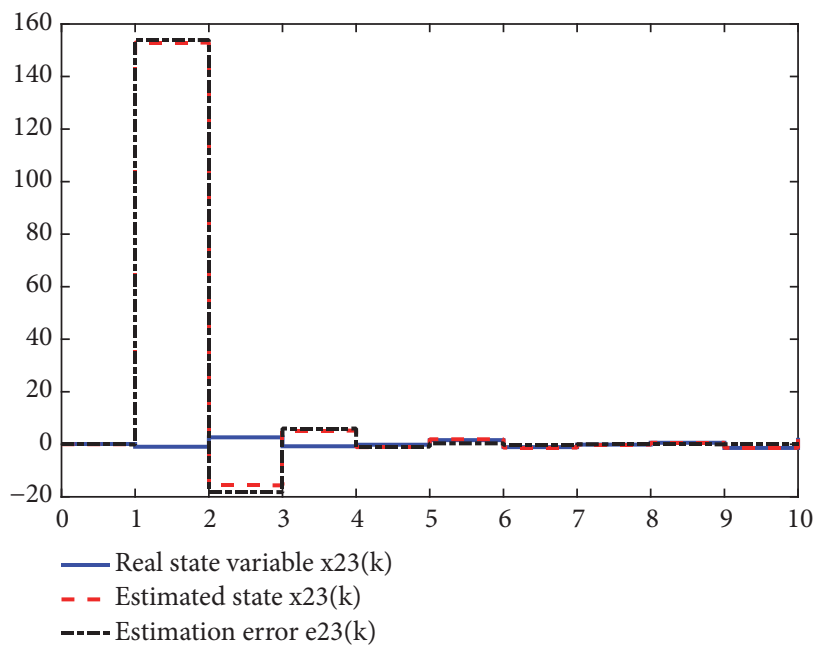

FIgURE 6: Real state $x_{23}(k)$, estimated state $\hat{x}_{23}(k)$, and estimation error $e_{23}(k)$. 


\section{Conflicts of Interest}

The authors declare that there are no conflicts of interest regarding the publication of this paper.

\section{References}

[1] X. Ye, "Decentralized adaptive stabilization of large-scale nonlinear time-delay systems with unknown high-frequency-gain signs," IEEE Transactions on Automatic Control, vol. 56, no. 6, pp. 1473-1478, 2011.

[2] B. Zhao, D. Wang, G. Shi, D. Liu, and Y. Li, "Decentralized control for large-scale nonlinear systems with unknown mismatched interconnections via policy iteration," IEEE Transactions on Systems, Man, and Cybernetics: Systems, vol. 48, no. 10, pp. 1725-1735, 2018.

[3] X. Yang and H. He, "Adaptive dynamic programming for decentralized stabilization of uncertain nonlinear large-scale systems with mismatched interconnections," IEEE Transactions on Systems, Man, and Cybernetics: Systems, pp. 1-13, 2018.

[4] L. N. Tan, "Distributed Hoo optimal tracking control for strictfeedback nonlinear large-scale systems with disturbances and saturating actuators," IEEE Transactions on Systems, Man, and Cybernetics: Systems, pp. 1-13, 2018.

[5] T. Takagi and M. Sugeno, "Fuzzy identification of systems and its applications tomodeling and control," IEEE Transactions on Systems, Man, and Cybernetics, Part B: Cybernetics, vol. SMC-15, no. 2, pp. 116-132, 1985.

[6] Z. Lendek, T. M. Guerrea, R. Babuska, and B. D. Schutter, Stability Analysis and Nonlinear Observer Design Using TakagiSugeno Fuzzy Models, Springer-Verlag, Berlin, Germany, 2010.

[7] K. Tanaka and H. O. Wang, Fuzzy Control Systems Design and Analysis: A Linear Matrix Inequality Approach, John Wiley \& Sons, Inc, New York, NY, USA, 2001.

[8] H. B. Zhang and G. Feng, "Stability analysis and Ho controller design of discrete-time fuzzy large scale systems based on piecewise lyapunov functions," IEEE Transactions on Systems, Man, and Cybernetics, Part B (Cybernetics), vol. 38, no. 5, pp. 1390-1401, 2008.

[9] C. Hua and S. X. Ding, "Decentralized networked control system design using T-S fuzzy approach," IEEE Transactions on Fuzzy Systems, vol. 20, no. 1, pp. 9-21, 2012.

[10] W.-J. Wang and W.-W. Lin, "Decentralized PDC for large-scale T-S fuzzy systems," IEEE Transactions on Fuzzy Systems, vol. 13, no. 6, pp. 779-786, 2005.

[11] W. Chang and W. J. Wang, "Ho fuzzy control synthesis for a large-scale system with a reduced number of LMIs," IEEE Transactions on Fuzzy Systems, vol. 23, no. 4, pp. 1197-1210, 2015.

[12] W. Ji, A. Wang, and J. Qiu, "Decentralized fixed-order piecewise affine dynamic output feedback controller design for discretetime nonlinear large-scale systems," IEEE Access, vol. 5, pp. 1977-1989, 2017.

[13] H. S. Kim, J. B. Park, and Y. H. Joo, "Decentralized sampleddata tracking control of large-scale fuzzy systems: an exact discretization approach," IEEE Access, vol. 5, pp. 12668-12681, 2017.

[14] T. Wang and S. Tong, "Decentralized control design for switched fuzzy large-scale systems with Ho performance," Neurocomputing, vol. 165, pp. 330-337, 2015.

[15] Z. Zhong, C.-M. Lin, Z. Shao, and M. Xu, "Decentralized eventtriggered control for large-scale networked fuzzy systems," IEEE Transactions on Fuzzy Systems, vol. 26, no. 1, pp. 29-45, 2018.
[16] K. Tanaka, H. Yoshida, H. Ohtake, and H. O. Wang, "A sum-ofsquares approach to modeling and control of nonlinear dynamical systems with polynomial fuzzy systems," IEEE Transactions on Fuzzy Systems, vol. 17, no. 4, pp. 911-922, 2009.

[17] A. Papachristodoulou, J. Anderson, G. Valmorbida, S. Prajna, P. Seiler, and P. A. Parrilo, "SOSTOOLS: sum of squares optimization toolbox for MATLAB," Version 3.01, 2016.

[18] K. Tanaka, H. Ohtake, T. Seo, M. Tanaka, and H. O. Wang, "Polynomial fuzzy observer designs: a sum-of-squares approach," IEEE Transactions on Systems, Man, and Cybernetics, Part B: Cybernetics, vol. 42, no. 5, pp. 1330-1342, 2012.

[19] C. Liu and H. K. Lam, "Design of a polynomial fuzzy observer controller with sampled-output measurements for nonlinear systems considering unmeasurable premise variables," IEEE Transactions on Fuzzy Systems, vol. 23, no. 6, pp. 2067-2079, 2015.

[20] H. K. Lam, "Stability analysis and control synthesis using membership function-dependent techniques," in Polynomial Fuzzy Model-Based Control Systems, Springer International Publishing, Switzerland, 2016.

[21] H. J. Kim, G. B. Koo, J. B. Park, and Y. H. Joo, "Decentralized sampled-data $\mathrm{H} \infty$ fuzzy filter for nonlinear large-scale systems," Fuzzy Sets and Systems, vol. 273, pp. 68-86, 2015.

[22] H. J. Kim, J. B. Park, and Y. H. Joo, "Decentralized Ho fuzzy filter for nonlinear large-scale sampled-data systems with uncertain interconnections," Fuzzy Sets and Systems, vol. 344, pp. 145-162, 2018.

[23] M. Mohamed, X.-G. Yan, S. K. Spurgeon, and B. Jiang, "Robust sliding-mode observers for large-scale systems with application to a multimachine power system," IET Control Theory \& Applications, vol. 11, no. 8, pp. 1307-1315, 2017.

[24] V. N. Phat, N. T. Thanh, and H. Trinh, "Full-order observer design for nonlinear complex large-scale systems with unknown time-varying delayed interactions," Complexity, vol. 21, no. 2, pp. 123-133, 2015.

[25] H. Wang and G.-H. Yang, "Decentralized fault detection for affine T-S fuzzy large-scale systems with quantized measurements," IEEE Transactions on Fuzzy Systems, vol. 26, no. 3, pp. 1414-1426, 2018.

[26] X.-L. Wang and G.-H. Yang, "Robust Ho filter design for discrete-time interconnected fuzzy systems with partially unknown membership functions and past output measurements," Neurocomputing, vol. 310, pp. 287-298, 2018.

[27] L. Zhang and H. Zhang, "Nonfragile Ho filtering for discretetime nonfragile ho filtering for discrete-time nonlinear interconnected systems nonlinear interconnected systems nonlinear interconnected systems," IFAC-PapersOnLine, vol. 49, no. 5, pp. 25-30, 2016.

[28] Z. Zhong, S. Fu, T. Hayat, F. Alsaadi, and G. Sun, "Decentralized piecewise Ho fuzzy filtering design for discrete-time largescale nonlinear systems with time-varying delay," Journal of The Franklin Institute, vol. 352, no. 9, pp. 3782-3807, 2015.

[29] Z. Zhong and Y. Zhu, "Observer-based output-feedback control of large-scale networked fuzzy systems with two-channel eventtriggering," Journal of The Franklin Institute, vol. 354, no. 13, pp. 5398-5420, 2017.

[30] W. Wang, W. Chang, and S. Yeh, "Unknown input based observer synthesis for uncertain Takagi-Sugeno fuzzy systems," IET Control Theory \& Applications, vol. 9, no. 5, pp. 729-735, 2015.

[31] A. Chibani, M. Chadli, and N. B. Braiek, "A sum of squares approach for polynomial fuzzy observer design for polynomial 
fuzzy systems with unknown inputs," International Journal of Control, Automation, and Systems, vol. 14, no. 1, pp. 323-330, 2016.

[32] M. Chadli, "An LMI approach to design observer for unknown inputs Takagi-Sugeno fuzzy models," Asian Journal of Control, vol. 12, no. 4, pp. 524-530, 2010.

[33] M. Chadli and H. R. Karimi, "Robust observer design for unknown inputs takagi-sugeno models," IEEE Transactions on Fuzzy Systems, vol. 21, no. 1, pp. 158-164, 2013.

[34] S. Guo, F. Zhu, and L. Xu, "Unknown input observer design for Takagi-Sugeno fuzzy stochastic system," International Journal of Control, Automation, and Systems, vol. 13, no. 4, pp. 1003-1009, 2015.

[35] A. Kruszewski, R. Wang, and T. M. Guerra, "Nonquadratic stabilization conditions for a class of uncertain nonlinear discrete time TS fuzzy models: a new approach," IEEE Transactions on Automatic Control, vol. 53, no. 2, pp. 606-611, 2008. 


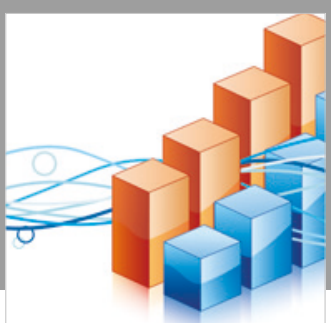

Advances in

Operations Research

\section{-n-m}
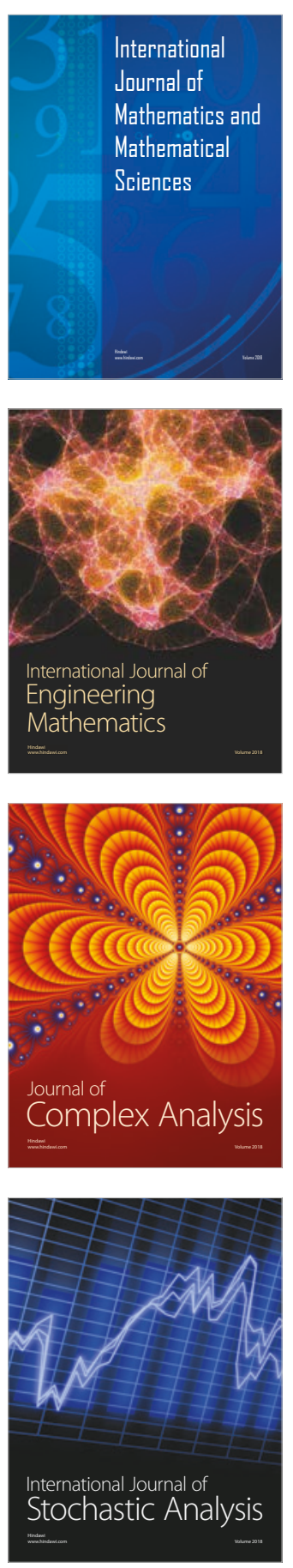
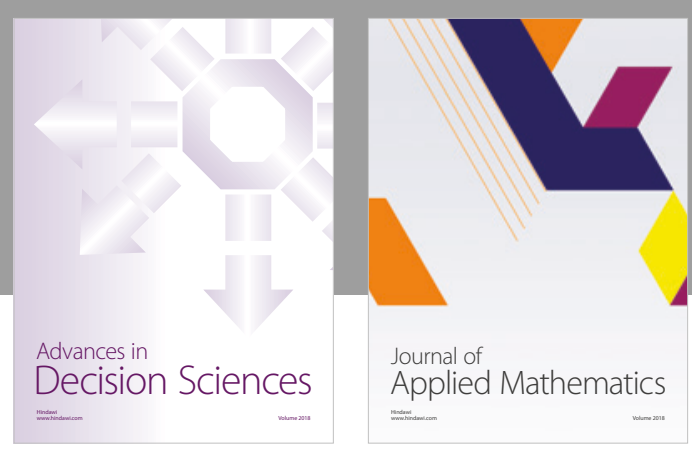

Journal of

Applied Mathematics
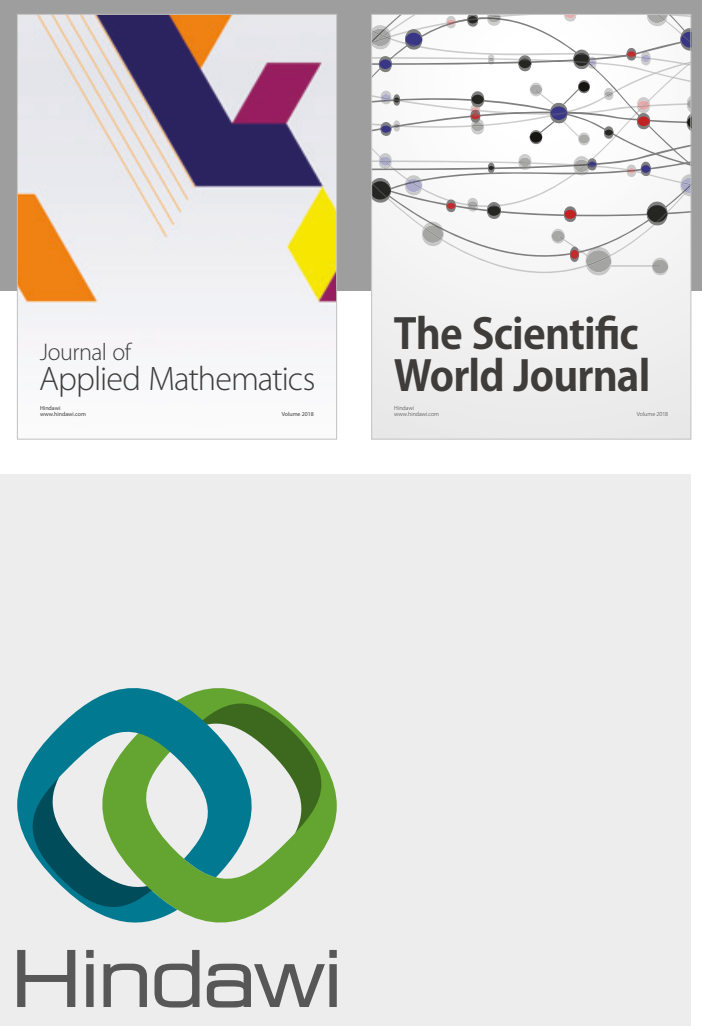

Submit your manuscripts at

www.hindawi.com

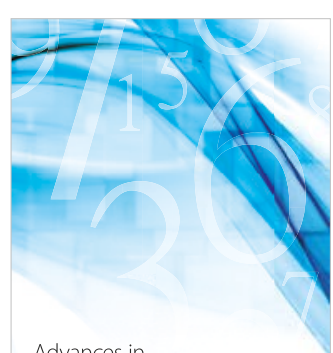

Advances in
Numerical Analysis
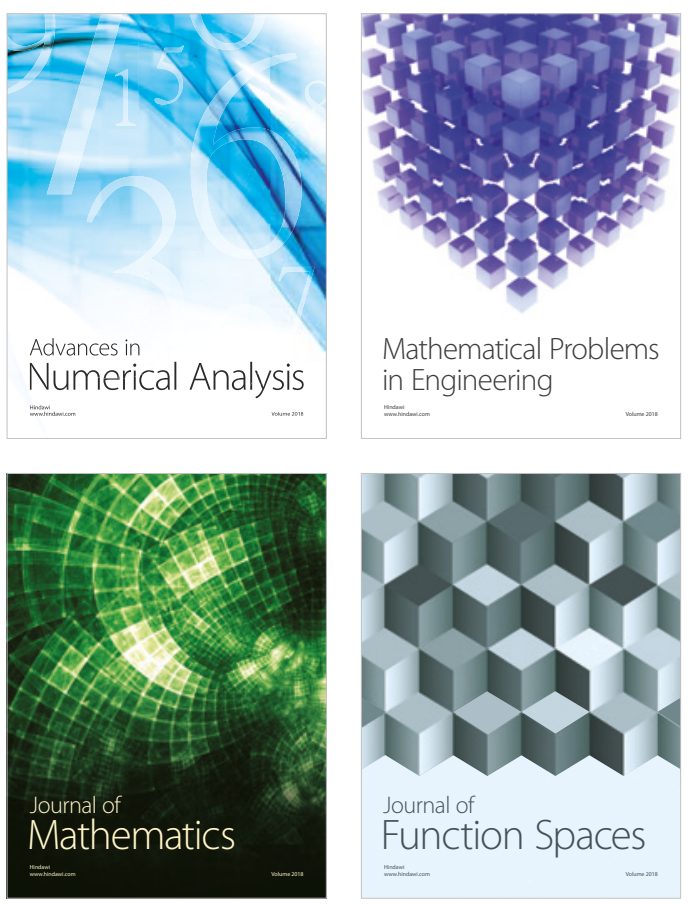

Mathematical Problems in Engineering

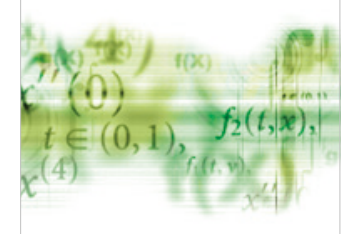

International Journal of

Differential Equations

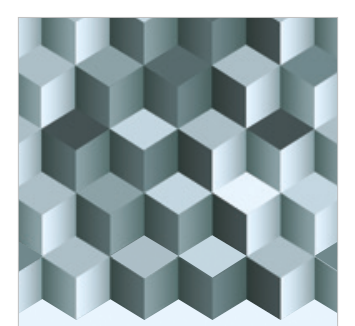

Journal of

Function Spaces

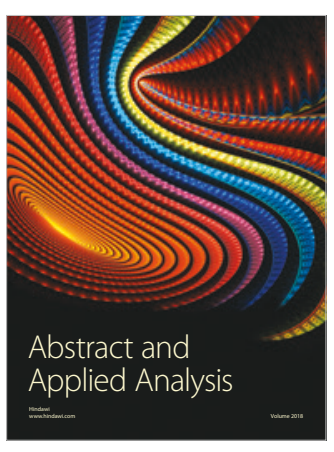

The Scientific

World Journal

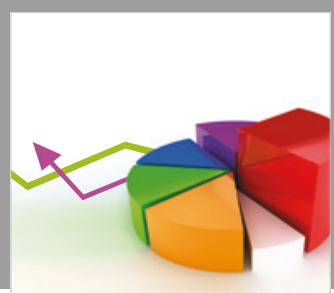

Journal of

Probability and Statistics
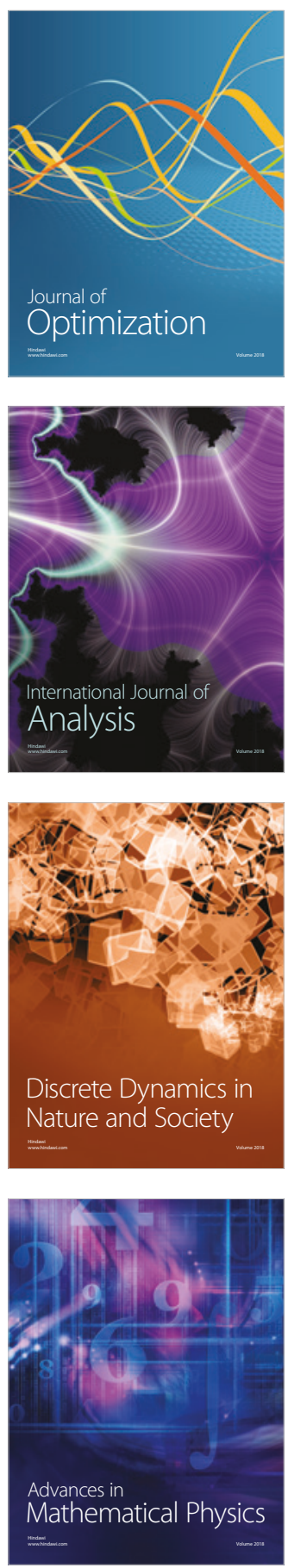